Musées, Patrimoine et Culture scientifiques et techniques

$143 \mid 2012$

septembre-octobre 2012

\title{
Compter la science
}

Jean-Charles Thuault et Sébastien Marie

URL : http://journals.openedition.org/ocim/1110

DOI : 10.4000/ocim. 1110

ISSN : 2108-646X

Éditeur

OCIM

Édition imprimée

Date de publication : 1 septembre 2012

Pagination : 28-35

ISSN : 0994-1908

Référence électronique

Jean-Charles Thuault et Sébastien Marie, « Compter la science », La Lettre de l'OCIM [En ligne],

143 | 2012, mis en ligne le 01 septembre 2014, consulté le 02 mai 2019. URL : http://

journals.openedition.org/ocim/1110; DOI : 10.4000/ocim.1110 


\section{Compter la science}

\section{Jean-Charles Thuault et Sébastien Marie *}

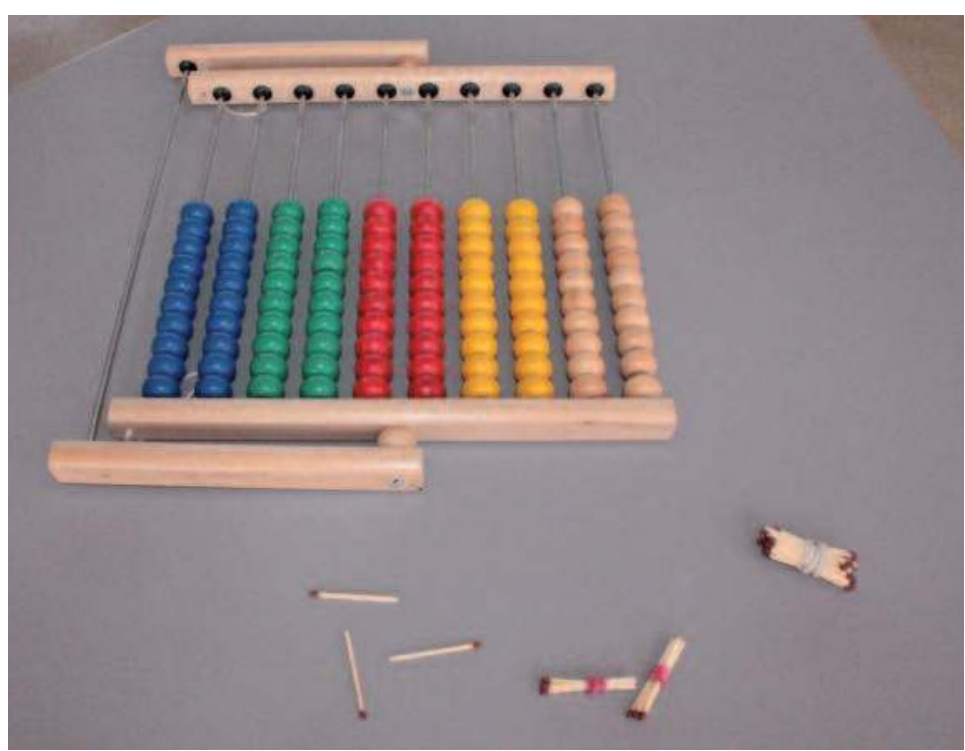

« Compter la science » : un boulier russe et des moutons comptés en base 4 . (C) Sébastien Marie

* Jean-Charles Thuault est directeur-adjoint d'ébulliScience ${ }^{\circledR}$ Sébastien Marie est coordinateur à ébulliScience ${ }^{\circledR}$ ebulliscience.lyon@gmail.com
Association lyonnaise dévolue à la diffusion de la culture scientifique et technique auprès du grand public, ébulliScience ${ }^{\circledR}$ mène actuellement en direction des scolaires de la maternelle au primaire un projet pour appréhender les mathématiques autrement: les responsables de "Compter la science » analysent les premiers résultats de cette opération ambitieuse et originale.

Des difficultés d'apprentissage en sciences et mathématiques (1) sont apparues dans le bilan des RRS (Réseaux de Réussite Scolaire) des quartiers ÉtatsUnis et Mermoz dans le $8^{\mathrm{e}}$ arrondissement de la ville de Lyon. C'est pourquoi ébulliScience ${ }^{\circledR}$, déjà implantée dans ces quartiers prioritaires, a proposé en 2010/2011 un nouveau projet portant sur le temps scolaire et intitulé «Compter la science », dans l'optique de sensibiliser, dès la maternelle, les enfants, les enseignants et les parents à la démarche scientifique ainsi qu'à l'importance des sciences et des mathématiques dans le parcours scolaire. L'idée est celle de faire des mathématiques, d'en faire autrement, et au-delà de transmettre le plaisir d'en faire et de compter, aussi bien pour les enfants que pour les enseignants. De tels projets existent déjà pour transmettre le plaisir de la lecture, il restait à adopter de tels objectifs dans le domaine des sciences et des mathématiques.

ébulliScience ${ }^{\circledR}$ a pour habitude de placer ses visiteurs en position d'être eux-mêmes les chercheurs. Cette pédagogie active est ici appliquée à de très jeunes enfants (de la grande section de maternelle au CE1) qui 
sont amenés à intégrer les étapes d'une véritable démarche d'investigation. Celle-ci doit rassurer l'enfant dans la formulation de ses hypothèses, lui permettant ainsi de construire lui-même son savoir à l'issue d'un processus d'essais-erreurs. À mesure qu'il expérimente, qu'il teste pour vérifier ses hypothèses, il comprend que l'erreur n'est pas grave et qu'elle fait même partie de son apprentissage. C'est à cette condition que l'élève trouvera la confiance en soi nécessaire à ses progrès, et principale source de sa motivation.

Au cœur de ce projet expérimental, se trouve également la volonté de donner du sens aux apprentissages en sciences et mathématiques. Ces disciplines sont fréquemment porteuses, dans les représentations, d'un côté abstrait, complexe et compliqué. L'association ébulliScience ${ }^{\circledR}$ entend montrer que les sciences et les mathématiques sont au contraire accessibles à tous, dès le plus jeune âge, et qu'elles permettent de répondre concrètement à des questions tout aussi concrètes. L'élève-chercheur construit son propre savoir en prenant conscience de la logique et du sens de l'évolution du savoir scientifique et mathématique construit au cours de l'histoire. Il s'agira de vérifier en quoi cette approche permet de faciliter les apprentissages.
Les objectifs poursuivis, ceux qui feront l'objet d'une évaluation, peuvent se résumer ainsi :

- permettre à tous, filles et garçons, de bénéficier d'un accès privilégié à l'apprentissage des sciences et mathématiques ;

- donner le goût pour les sciences et les mathématiques dès l'école maternelle ;

- faciliter la liaison entre temps de l'enfant et temps de l'élève ;

- soutenir les équipes éducatives par un apport d'expertise ; - soutenir les parents dans leur rôle éducatif en leur donnant accès à des outils pédagogiques.

\section{Une pédagogie active}

ébulliScience ${ }^{\circledR}$ intervient depuis 2010/2011 auprès de 10 classes appelées à être suivies sur 3 ans. La première année, ce sont 155 élèves de Grande Section qui en ont bénéficié, répartis dans 4 écoles maternelles des quartiers (Louis Pasteur, Jean Giono, Édouard Herriot et Olympe de Gouges). Chacune de ces classes bénéficie de 18 séances par an, réparties en 3 périodes de 6 semaines, conçues et animées par Aurélia Quillon, docteur en mathématiques. Un travail en demie classe sur des séances adaptées aux capacités d'attention d'enfants de cet âge signifie de bonnes conditions d'apprentissage.

\section{L’associotion ébulliscience ${ }^{\circledR}$}

Créée en 1998 avec le soutien de la communauté scientifique, l'association ébulliScience ${ }^{\circledR}$ est un opérateur reconnu par l'État et la collectivité pour sa contribution au développement de la culture scientifique et technique pour le grand public. Le visiteur n'y est pas considéré comme un «profane » qu'il s'agirait d'initier au savoir des spécialistes mais comme un être doué d'intelligence capable de se placer du côté de la science en train de se faire...

Et la science n'en finit pas de ne pas savoir ! C'est sur l'intelligence en action, en tant que bien commun, qu'ébulliScience ${ }^{\circledR}$ met un accent quasi exclusif afin qu'au-delà de l'inaccessible accumulation du savoir, la science soit perçue prioritairement comme une dynamique enthousiasmante.

ébulliScience ${ }^{\circledR}$ dispense des activités éducatives sur 2 sites permanents accueillant le public :

- à Vaulx-en-Velin, la salle de découvertes scientifiques, espace muséographique, accueille plus de 10000 visiteurs par an depuis son inauguration en mars 1999 par Georges Charpak ;
- à Lyon, l'Espace ébulliScience-Lyon 8 e reçoit 60 écoles par an depuis 2004 en séjours « Classes Sciences », ainsi que des stagiaires durant les vacances scolaires.

ébulliScience ${ }^{\circledR}$ dispose également depuis 2008 d'un dispositif d'Ateliers itinérants de Découvertes des sciences, avec le soutien de la Région Rhône-Alpes. Il permet d'organiser des activités temporaires sur les sites des partenaires éducatifs.

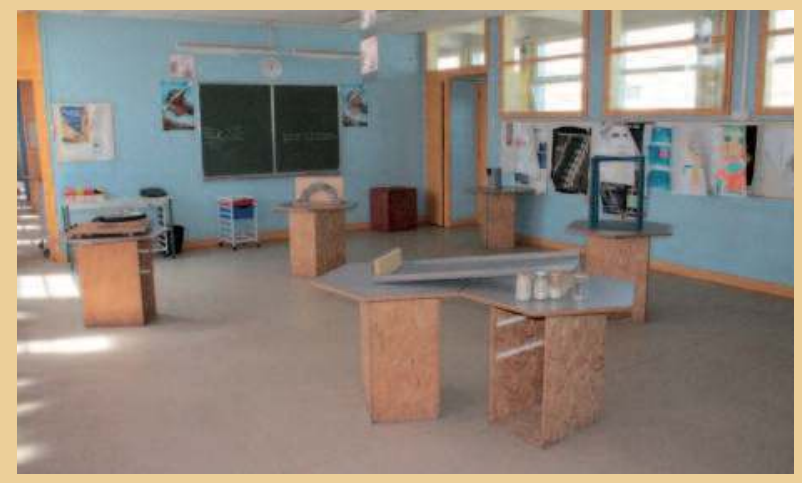

Une salle de découvertes scientifiques à ébulliScience ${ }^{\circledR}$ ( ) Sébastien Marie 


\section{Une initiation à la démarche d'investigation}

La première période consiste en une initiation à la démarche d'investigation. Des activités de découvertes scientifiques sont proposées aux élèves, choisies aussi bien pour attiser la curiosité que pour rappeler des objets du quotidien. Il est particulièrement important que les enfants puissent facilement parler à la maison de ce qu'ils ont fait en classe, et pourquoi pas de refaire certaines expériences avec leurs parents. Chacune des séances traite d'un thème différent, l'objectif prioritaire pour ces enfants de maternelle étant de pratiquer la démarche d'investigation, et non de développer des connaissances précises sur un thème particulier. Le lien, le fil d'Ariane entre toutes ces séances doit être la démarche d'investigation.

Les élèves sont amenés à mettre en question le monde qui les entoure, et à entrer dans une véritable démarche de chercheur, c'est-à-dire observer, émettre des hypothèses, tester par l'expérimentation, et en dégager eux-mêmes des conclusions. Les moments de manipulations sont couplés à des temps, essentiels, d'échanges et de dialogue entre élèves et animateur. Ces temps permettent de développer les capacités d'écoute et de discussion ; mais aussi, pour chaque enfant, une valorisation individuelle en s'exprimant devant le groupe ; et enfin, d'introduire progressivement un vocabulaire scientifique adapté aussi bien à leur âge qu'à une description précise des phénomènes. Ces temps sont également utiles pour que les élèves reformulent ce qu'ils ont découvert par euxmêmes durant la séance : ils doivent pouvoir prendre conscience de ce qu'ils ont appris.

À la fin de cette première période, chaque élève doit être capable de se poser des questions et d'y apporter de manière autonome des réponses. Tous ont un premier vécu de chercheur : il peut se tromper et tout de même apprendre quelque chose ; et ce qu'il apprend, il le doit à lui-même parce que personne ne lui a donné la réponse. Le droit à l'erreur et la valorisation personnelle légitimée par les découvertes de l'enfant ne sont pas des «allant-de-soi » et méritent d'être régulièrement signalés. En effet, il s'agit de pouvoir développer un rapport ludique au savoir, plus propice aux apprentissages, donner l'envie et le goût d'apprendre.

\section{Compter?}

Depuis que l'homme vit en société, il a eu besoin de compter pour répondre à des besoins vitaux : compte du nombre de membres de la tribu, des réserves de nourriture, des troupeaux... L'histoire des nombres coïncide avec celle de l'écriture : lorsque l'Homme a appris à parler, il a aussi appris à compter; et lorsque l'Homme a appris à écrire, il a appris à calculer. Or, cette histoire s'étale sur plus de 10000 ans et si les enfants apprennent assez rapidement à parler, ils éprouvent souvent plus de mal à apprendre à compter avant l'entrée à l'école élémentaire à 6 ans.

Afin de permettre à l'enfant de mieux appréhender le nombre, nous l'amenons au cours de cette deuxième période à découvrir l'histoire des nombres tels que nous les connaissons. Des voyages dans le temps et/ou dans l'espace sollicitent un imaginaire et donnent des repères propres à l'activité qui facilitent grandement la mémorisation des points abordés au cours des séances. Par ailleurs, reproduire à l'échelle de l'enfant la très longue histoire des nombres a pour objectif de faire ressentir les logiques d'évolution, les besoins de complexification et surtout de dévoiler le sens des nombres pour en favoriser chez l'enfant la construction.

Il s'agit de retrouver les solutions pour compter, depuis le simple comptage visuel primitif permettant de dénombrer jusqu'à 4 , jusqu'à des méthodes d'appareillement, en passant par le comptage corporel. Pour chaque solution de comptage trouvée par les enfants, l'animatrice leur indique sur une carte du monde les civilisations ayant employé cette méthode. De nombreux jeux sont sollicités aussi bien pour que les élèves prennent plaisir à compter que pour leur faire prendre conscience du caractère concret du nombre et leur en faire saisir tout le sens. Ce sont autant de moteurs pour la suite de leurs apprentissages. Le nombre doit prendre un sens réel pour les

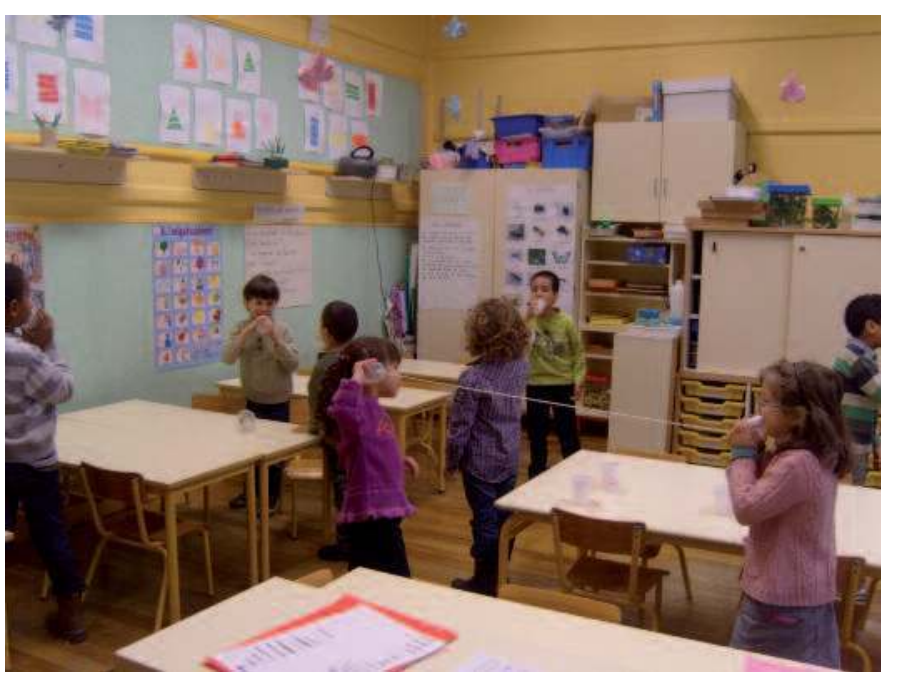

Des élèves de Grande Section découvrent la démarche expérimentale avec des «Ficellophones». (c) Aurélia Quillon 
enfants, avant que leur soit proposé un passage au nombre abstrait quand celui-ci est associé à diverses représentations.

À la fin de cette période, tous les enfants doivent être en mesure d'utiliser une méthode parmi toutes celles abordées (comptage visuel, cordelette à nœuds, bâton à entailles, comptage sur le corps, comptage sur les mains...) pour compter de manière concrète au moins jusqu’à 30 .

\section{Calculer}

Une fois les nombres acquis, l'animatrice propose aux enfants de les manipuler, toujours de manière concrète, pour réaliser leurs premiers calculs. Des additions et soustractions sont réalisées par les enfants sur de petites quantités d'objets.

Pour pouvoir aborder le calcul avec des nombres abstraits tel qu'il sera introduit à l'école élémentaire, l'enfant doit maîtriser l'écriture de position des nombres. En plus des nombres, cette écriture implique d'utiliser une base. Pour que les enfants puissent découvrir le concept de base, il leur est demandé de trouver, en petits groupes, une manière de dénombrer une grande quantité d'objets. Très vite, les enfants sont contraints de regrouper les objets en «paquets » et " paquets de paquets ». La taille des paquets, ou base, n'est pas imposée par l'animatrice, mais pourra varier d'un groupe d'enfants à l'autre. Une synthèse des solutions proposées par les élèves permet de discuter des avantages et inconvénients de chaque base. En s'appuyant sur une carte du monde, l'animatrice expose les bases utilisées par les différentes civilisations.

Une fois le principe de la base établi, les enfants sont amenés à réfléchir à des solutions pour écrire les nombres. Dans un premier temps, les enfants imaginent un grand nombre de symboles différents pour les unités et chaque multiple de la base sans que la valeur d'un nombre soit liée à sa position. L'animatrice présente quelques systèmes de numérations additives dont les chiffres possèdent chacun leur valeur propre, indépendamment de leur position. Devant le très grand nombre de symboles nécessaires pour représenter même de petites quantités, l'animatrice propose de chercher une solution plus simple. Pour accompagner les enfants dans leur recherche de simplification, l'animatrice peut présenter un ou plusieurs exemples de numérations hybrides utilisant un nombre plus réduit de symboles pouvant être supprimés pour arriver aux numérations de positions.
Les enfants apprennent à écrire des nombres à la manière des Mayas, des Chinois, des Égyptiens, et de manière moderne. Les nombres proposés à l'écriture ne nécessitent pas, dans un premier temps, l'utilisation d'un zéro. Les enfants sont invités, dans un second temps, à proposer leurs propres nombres. La nécessité du zéro peut alors apparaître dans ce cadre.

\section{Compter la Science en CP}

Les mêmes classes, en CP désormais, sont suivies en 2011/2012 pour trois nouvelles périodes de travail.

Investigations autour des liquides et des solides Comme lors de la première période de l'année précédente, la programmation débute par le versant scientifique du projet. Les élèves poursuivent leur apprentissage de la démarche d'investigation, mais autour cette fois-ci d'un même thème filé sur les séances : les liquides et les solides. L'objectif est de... consolider, précisément, l'utilisation des étapes de la démarche d'un chercheur par les élèves en leur permettant de sentir leur progression d'une séance à l'autre dans leur construction de connaissances nouvelles sur ce thème figurant aux programmes.

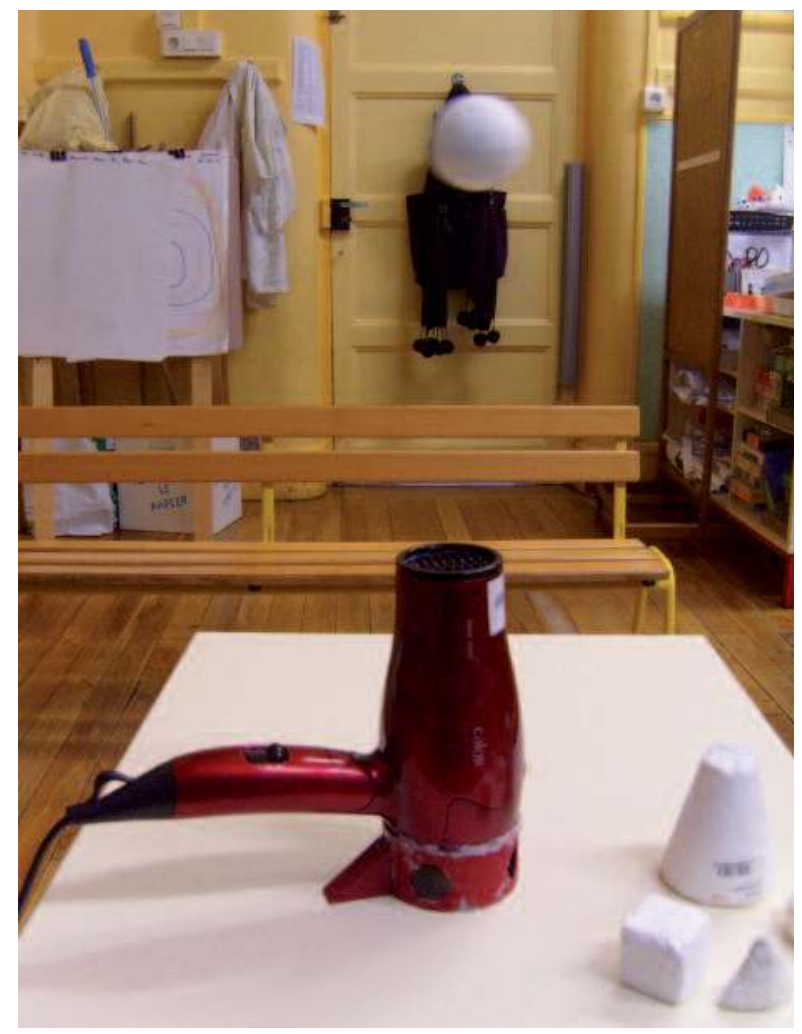

Les élèves font jongler des objets avec l'air et se posent la question «Qu'est-ce qui compte? » (expérience « Jongl'air »). ๑) Aurélia Quillon 
Les temps d'échanges et de restitution, en fin de séance et en début de séance suivante, sont toujours privilégiés, mais une plus grande structuration de ces comptes-rendus par les élèves est attendue, notamment en demandant un récit chronologique témoin de la progression logique des questionnements. Une synthèse ainsi qu'un dessin de la séance, destinés à un « cahier de chercheur » assurant le lien avec les familles, sont faits en classe pour permettre d'échanger entre élèves, enseignant et animatrice.

En termes de connaissances, les expérimentations menées par les élèves leur permettent de découvrir des différences et des similitudes entre les liquides et les solides. La première étape est bien entendu celle de pouvoir définir ce qu'est un liquide et ce qu'est un solide. Différentes propriétés des solides et des liquides sont ensuite testées par l'expérimentation.

\section{Les machines à calculer}

En grande section, les enfants ont découvert de multiples manières de compter. L'immense majorité a pris du plaisir à manipuler les nombres. Lors de la $3^{\mathrm{e}}$ période de la première année, les enfants se sont interrogés sur la manière de concevoir et d'écrire de grands nombres (supérieurs à 100). Ils ont été sensibilisés au principe de la base.

\section{Un exemple de séonce : Le comploge primiliff}

\section{Accueil}

L'animatrice salue les enfants et les incite à se remémorer ce qu'ils ont découvert en tant que chercheurs lors des séances précédentes, ainsi que la démarche d'investigation qui a permis ces découvertes. La séance peut débuter :

\section{Introduction}

"Aujourd'hui, nous allons faire des expériences pour découvrir comment les hommes qui vivaient à la préhistoire comptaient. Pour cela, nous allons faire un voyage dans le temps. Vous êtes prêts? On embarque. Imaginez que vous êtes il y a très longtemps (avant les rois et les chevaliers, avant les Romains et les Gaulois, avant les Pharaons).Vous êtes des hommes et des femmes préhistoriques! Vous êtes à l'époque de l'âge de glace, avec les mammouths. Vous ne savez pas parler, pas écrire et évidemment vous ne savez pas compter. Comment faire pour vous compter? Quand vous partez à la chasse au mammouth, comment faire pour savoir si tous les chasseurs sont rentrés ou s'il en manque?»

Première expérience : «Un, deux, trois, quatre, beaucoup "

«À la Préhistoire, vous ne pouvez pas compter (1, 2, 3, $4,5, \ldots)$, mais vous powvez regarder et essayer de dire combien il y a d'objets. Par exemple, si je vous montre un seul bâton, vous powvez me dire qu'il y en a un. Vous n'avez pas besoin de compter, vous le voyez tout de suite. Avec deux bâtons ?... Ça marche aussi! Mais si je pose un tas de bâtons, vous ne pouvez pas me dire combien il y en a». Les enfants jouent avec des jeux de cartes et essayent de voir combien d'objets sont dessinés sur les cartes. Ensemble, ils arrivent à la conclusion qu'ils sont capables de voir jusqu'à 4 .
Au-dessus, on ne peut pas savoir sans compter. On peut simplement dire qu'il y en a beaucoup. On appelle cela le comptage visuel primitif. L'animatrice leur explique que c'est pareil dans le monde entier et leur donne des exemples à travers le monde.

\section{Deuxième expérience : Correspondance unité par unité}

Retour à la Préhistoire. L'animatrice fait remarquer aux enfants qu'ils forment une tribu avec plus de 4 personnes. Ils peuvent donc dire qu'ils sont beaucoup mais ne savent pas combien. Dans ce cas, comment faire pour savoir si tous les chasseurs sont bien rentrés de la chasse au mammouth ?

Avec la complicité de l'animatrice, les enfants sont invités à jouer à la chasse au mammouth :

- installation de la tribu sur les chaises : une chaise par membre de la tribu ;

- départ à la chasse ;

- retour : tout le monde est-il là ? Oui. Tant mieux ; - on y retourne! Le mammouth-animatrice attrape deux chasseurs.

- retour : tout le monde est-il là ? Non ;

- l'animatrice demande aux enfants comment ils ont remarqué qu'il manquait des chasseurs. Réponse : Il reste des chaises vides.

\section{Conclusion}

"En comparant le nombre de chaises et le nombre d'enfants, vous avez pu savoir si tout le monde était présent. C'est une comparaison. Il suffit de se donner un repère qui ne change pas (ici les chaises), et on peut compter un groupe. Les mathématiciens appellent cela la correspondance unité par unité ou la bijection ». 
Cette année, un retour au « Pays de 10 » rappelle et consolide la compréhension du principe de la base. L'histoire des nombres est de nouveau sollicitée, avec les chiffres romains élaborés par les bergers qui comptaient comme au «Pays de 10 ». Outre l'avantage de contribuer à l'imaginaire collectif en poursuivant les voyages et retrouvant des rôles déjà joués, refaire l'histoire de l'écriture des nombres donne son plein sens à la notation positionnelle que nous utilisons. Ressentir les inconvénients des systèmes successifs, imaginer des solutions aux problèmes posés, c'est mieux comprendre pourquoi aujourd'hui, nous écrivons les nombres de cette façon, et ce qu'ils signifient.

Ayant compris les nombres, les enfants vont être invités lors de cette deuxième période à les manipuler et à réaliser des calculs. Afin de comprendre la signification des opérations, les enfants vont tout d'abord réaliser de manière concrète des additions et soustractions sur de petites quantités d'objets.

Une fois la signification des opérations acquise, les enfants vont réaliser leurs premiers calculs, toujours de manière concrète, à l'aide de différentes machines à calculer. Les enfants vont être amenés à utiliser différents outils de calcul : la règle à calculer et le boulier russe. Le boulier russe permet d'écrire et de manipuler des nombres en utilisant une numération de position en base 10. Des additions simples puis des additions avec retenues peuvent être réalisées, rendues réelles par le déplacement des boules sur le boulier, ce qui est un moyen de donner du sens à de grands nombres ainsi que de comprendre de manière concrète la signification d'une addition, d'une soustraction et des retenues lors de ces opérations. Des concours ludiques sont organisés sur le boulier pour renforcer encore le plaisir pris à calculer.

La finalité de cette période est que les enfants découvrent et s'approprient, grâce à une manipulation concrète, les opérations sur les nombres. L'objectif est que chaque enfant prenne confiance dans ses capacités à compter et à calculer pour aborder sereinement les apprentissages de l'école élémentaire.

Et la suite...

Une troisième période en $\mathrm{CP}$ aura pour objectif de consolider toutes ces acquisitions sur les nombres et les calculs. Et les mêmes classes seront suivies en 2012/2013, en CE1, pour clore ce projet expérimental. Il s'agira alors de pouvoir évaluer les apports d'une telle programmation pédagogique en regard du diagnostic initial.

\section{Une démarche partenariale}

Ce projet a été pensé dans une démarche partenariale avec le Projet Éducatif Lyonnais et la circonscription de Lyon $8^{\text {e }}$ de l'Éducation nationale, pour s'intégrer dans un Projet Global d'Activités Scientifiques. L'objectif est certes de donner le goût aux plus jeunes élèves de faire des mathématiques, mais également de le donner aux enseignants.

À ce titre, le projet comporte un volet de formation des enseignants, par le moyen de conférences pédagogiques pour chacune des périodes. ébulliScience ${ }^{\circledR}$ y présente les contenus qui seront abordés en mettant les enseignants en situation. Ils sont formés, et mis en capacité d'appliquer la démarche d'investigation avant que leurs élèves ne le soient. Ils sont également sensibilisés à l'histoire des nombres. Pour compléter la formation, des temps d'accompagnement sont prévus autour des séances, consacrés à des échanges pédagogiques sur ce qui peut être réinvesti en classe.

Le projet «Compter la science » prend tout son sens à partir du moment où l'enseignant qui a bénéficié de l'intervention dans sa classe se trouve en mesure de proposer la même chose de manière autonome les années suivantes. Connaissances et méthodes pédagogiques doivent être transmises en même temps que l'intérêt et le goût pour les contenus abordés. Certaines séances sont également consacrées à la réalisation de bouliers ou règles à calculer destinées à être réutilisées en classe par l'enseignant.

Ce projet vise également à établir un partenariat éducatif avec les parents. "Compter la science » a en effet connu un prolongement dans le cadre d'un projet de circonscription et en lien avec la « Mission Égalité » de la Ville de Lyon. Les statistiques montrent d'une part que les habitants des quartiers prioritaires ont moins accès aux études scientifiques, et d'autre part que les filles ont moins accès à ces mêmes études que les garçons. Partant du principe qu'il n'y avait pas de raison objective à cette double discrimination, ébulliScience ${ }^{\circledR}$ s'est proposé d'organiser des rencontres-débat dans chacune des écoles concernées par le projet « Compter la science ». L'idée était de mettre les parents en situation d'être des chercheurs, comme leurs enfants, pour prendre conscience que chacun peut faire des sciences et des expériences en famille. Par la même occasion, cette action permet aux parents de mieux connaître et comprendre ce qui est demandé à leurs enfants à l'école. Quant au débat, il visait à échanger sur le rôle 


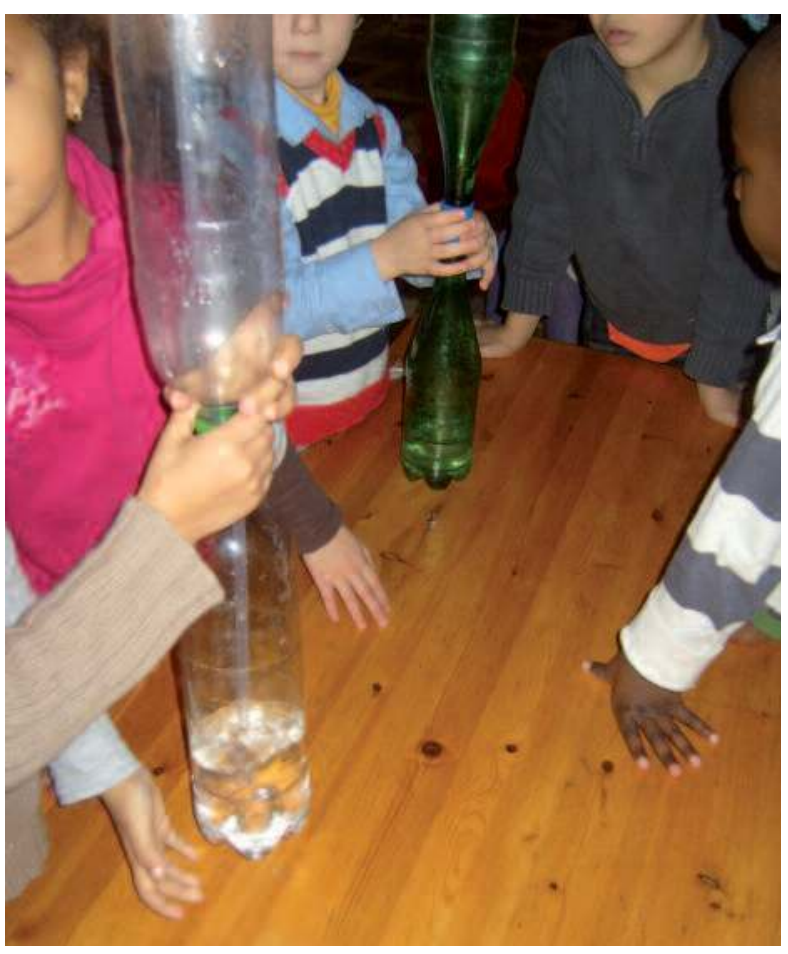

"Comment fait l'eau de cette bouteille pour jaillir en fontaine?" (c) Aurélia Quillon

des sciences dans la scolarité, et sur les représentations des parents vis-à-vis des sciences, et finalement de l'école en général, pour leurs enfants.

Une première rencontre a eu lieu à l'école Pasteur en décembre 2011, réunissant plus de 30 parents témoignant d'un vif intérêt pour la démarche et d'une véritable attente d'actions scientifiques dont pourraient bénéficier leurs enfants.

\section{Une démarche d'évaluation}

ébulliScience ${ }^{\circledR}$ s'inscrit dans une démarche d'évaluation globale. Depuis le diagnostic élaboré par l’Éducation nationale d'un déficit d'apprentissages en sciences et mathématiques dans les quartiers concernés, il s'agissait de proposer des actions susceptibles d'y remédier. Lors des différentes étapes du projet il est essentiel de pouvoir juger du niveau d'atteinte des objectifs fixés. ébulliScience ${ }^{\circledR}$ a en conséquence fixé des critères d'évaluation.

Le premier point est relatif au plaisir pris par les enfants à faire des sciences et des mathématiques. Les enseignants témoignent d'une grande envie de leurs élèves de raconter ce qu'ils ont fait. Nombreux sont les enfants qui ont refait chez eux une expérience avec leurs parents $(50 \%)$. La plupart des enfants sont en demande de ces séances hebdomadaires. Il est clair que cette motivation ne peut que faciliter l'acquisition des contenus, également mesurée.

Au bout de deux séances, $90 \%$ des élèves sont capables d'observer une expérience et d'émettre des idées en rapport avec elle. Tous comprennent le mot « hypothèse » et $95 \%$ d'entre eux ont appris au moins quatre termes scientifiques nouveaux. Tous les élèves ont acquis les repères chronologiques et la plupart des repères géographiques liés à la longue histoire des nombres. $90 \%$ d'entre eux se révèlent capables d'utiliser l'une des méthodes de comptage jusqu'à 30 étudiée (même si tous ne maîtrisent pas le vocabulaire pour nommer les nombres jusqu'à 30 ) et $80 \%$ en maîtrisent au moins deux.

Enfin, des apprentissages transversaux ont été permis par ces séances. Une grande progression du nombre d'élèves qui prennent la parole est constatée au fil de l'année (de 60 \% à $90 \%$ ). C'est plus flagrant encore lorsqu'il s'agit de prendre la parole pour exprimer une idée (de $25 \%$ à $65 \%$ ). Cela témoigne d'une confiance en soi accrue pour la plupart des élèves, et du développement de leurs compétences langagières pour s'exprimer et écouter les autres. Toutefois, il est à signaler que certains élèves rencontrent des difficultés pour exprimer oralement ce qu'ils ont compris, mais peuvent très bien y parvenir par le moyen du dessin. En effet, un dessin de l'expérience est demandé à la fin de chaque séance, et si seulement $15 \%$ des élèves sont capables de produire un dessin en rapport avec l'expérience en début d'année, il en va ainsi pour $80 \%$ d'entre eux lors des dernières séances, avec des dessins schématiques de plus en plus construits.

L'implication des enseignants est essentielle dans le projet, et ébulliScience ${ }^{\circledR}$ note que tous sont capables de réinvestir la démarche d'investigation et sont sensibilisés à l'histoire des nombres qui leur ont été présentées en conférence pédagogique. Tous, également, reprennent en classe le travail effectué pendant "Compter la science », que ce soit par des temps de discussion, avec ou sans l'animatrice, une dictée de texte à la maîtresse...

\section{Conclusion}

À mi-parcours de ce projet expérimental, les premières évaluations sont encourageantes en regard des objectifs visés. Une évaluation rigoureuse permet de juger de l'efficacité de la méthode en comparant les niveaux des classes suivies à d'autres classes de ces mêmes écoles n'ayant pas participé à "Compter la science ». 
